\title{
An Integral Treatment For Coupled Heat and Mass Transfer By Natural Convection From A Radiating Paraboloid In A Porous Medium
}

\author{
N. Bano and B. Singh \\ Department of Mathematics, Dr. Babasaheb Ambedkar Technological University, Lonere \\ \{snasreenbano@yahoo.in ; bbsingh@dbatu.ac.in \}
}

\begin{abstract}
In the present paper, an analytical study of the effects of radiation on the buoyancy induced heat and mass transfer by natural convection from an isothermal paraboloid embedded in a saturated porous medium has been done in case of constant temperature and concentration. For the analysis of the problem, an integral method of Von Karman type has been used. The governing parameters for the problem under study are the buoyancy ratio $(N)$, Lewis number $(L e)$ and radiation parameter $\left(R_{d}\right)$. The computed results have covered a wide range of the governing parameters. It has been concluded that the local Nusselt number decreases while the local Sherwood number increases for aiding flow for increasing Lewis number. But an opposite trend is observed for opposing flow. The local Nusselt number decreases while the local Sherwood number increases for increasing values of the radiation parameter in case of aiding flow.. An opposite trend is observed for opposing flow.
\end{abstract}

Keywords: Buoyancy, Integral method, Heat transfer, Natural convection, Boundary layer thickness

\section{Introduction}

The study of coupled heat and mass transfer due to buoyancy and radiation effects in saturated porous media is of considerable interest due to its energy-related engineering and geophysical applications such as thermal insulation of buildings, enhanced recovery of petroleum resources, filtration process, groundwater pollution, etc. One problem of special interest is when the intrusive magma is trapped in an aquifer such that the free convection in the ground-water is generated adjacent to the hot intrusion. Due to the concentration gradient between the intrusion and ground water, the mass transfer wall occurs simultaneously.

On account of the afore-mentioned facts only, Bejan and Khair [1] were the first researchers to report a systematic study of heat and mass transfer along a wall embedded in a saturated porous medium with constant temperature and concentration. For the general case of an axi-symmetric body of arbitrary shape, however, the literature is still very scanty. Only a few authors [2-3] have made investigations to report heat transfer related results.

From the fundamental perspectives, Nield [4] made the first attempt to study the stability of the convective flow in horizontal layers with imposed temperature and concentration gradients. This was then followed by Khan and Zebib [5] in the study of flow stability in vertical porous layer. Trevisan and Bejan [6-8] have also conducted a series of investigations of these effects on natural convection for various geometries. Yücel [9] investigated the problem of heat and mass transfer along vertical surface embedded in saturated porous media. Lai et al. [10] studied the heat and mass transfer by natural convection from slender bodies of revolution embedded in porous media. Lai and Kulacki [11] generalized the problem tackled by Bejan and Khair [1] and obtained the similarity solutions of the boundary layer equations adjacent to at vertical surfaces with variable wall temperature and concentration, together with aiding buoyancies. Nakayama and Hossain [12] and Singh and Queeny [13] obtained integral solutions for problems for aiding buoyancies adjacent to vertical surfaces. Angirasa et al. [14] obtained the finite-difference solutions for natural convection with opposing buoyancy effects in a fluid saturated porous medium. Amahmid et al. [15] presented a numerical study for buoyancy layer type flows in a vertical porous enclosure induced by opposing buoyancy forces. Yih [16] studied the heat and mass characteristics of natural convection about a truncated cone embedded in a saturated porous medium. Chamakha [17] focused on the study of coupled heat and mass transfer by natural convection about a truncated cone in the presence of magnetic field and radiation effects. Bansod et al. [18] investigated the heat and mass transfer by natural convection from a vertical surface to a stratified Darcian fluid. Bansod [19] studied the Darcy's model of buoyancy layer flows in a horizontal porous medium induced by combined buoyancy forces.

B. Iyer, S. Nalbalwar and R.Pawade(Eds.)

ICCASP/ICMMD-2016. Advances in Intelligent Systems Research.

Vol. 137, Pp. 661-670.

(C) 2017- The authors. Published by Atlantis Press

This is an open access article under the CC BY-NC license (http://creativecommons.org/licens)es/by-nc/4). 
Singh and Chandarki [20] studied the coupled heat and mass transfer by natural convection from a vertical cylinder embedded in a saturated porous medium. The objective of the present paper is to extend the work of Singh and Chandarki [20] by taking into consideration the radiative properties of the fluid. To analyse the problem, an integral approach of Von Karman type has been used. Most of the problems governing various flow-fields in fluid mechanics are non-linear. So, it becomes significant to develop efficient methods to solve them. Ever since the advent of high speed computers, the numerical techniques for finding the solutions of highly non-linear differential equations have also been developing very quickly. However, it is still very difficult task to obtain the analytic approximations of these equations, despite the availability of much higher quality symbolic computation software such as MATHEMATICA, MATLAB, Maple, NAG, and so on. The reason might be that we do not have a satisfactory analytic tool valid for finding the solutions of problems with stronger non-linearity. The perturbation techniques are also not applicable in all the cases, for they are essentially based on the existence of small/large parameters, called the perturbation quantities, in the equations governing the flow-fields. The absence of such perturbation quantities largely restricts the application of these perturbation techniques.

The integral technique of Von Karman type, on the other hand, is different from the perturbation techniques in the sense that it is applicable even for non-linear problems where the governing equations and/or boundary conditions do not contain any small/large parameters at all. This method with great freedom and precision provides highly accurate approximations to non-linear problems. On account of this reason only, Nakayama and Hossain [12], Singh and Queeny [13], Bansod et al. [19], Singh and Chandarki [20] and Singh and Sharma [21] have successfully applied this integral approach to find analytical solutions of equations governing the combined heat and mass transfer by natural convection in porous media for various geometries.

The radiative effects have important applications in physics and engineering. The radiation heat transfer effects on different flows are very important in space technology and high temperature processes. But, a very little attention has been paid towards investigating the effects of radiation on boundary layers. The thermal radiation effects play an important role in controlling heat transfer in polymer processing industry where the quality of the final product depends on the heat controlling factors to some extent. High temperature plasmas, cooling of nuclear reactors, liquid metal fluids, magnetohydrodynamic accelerators, power generation systems are some important applications of radiative heat transfer from a vertical wall to conductive gray fluids. Recent developments in hypersonic flights, missile re-entry, rocket combustion chambers, power plants for inter-planetary flights and gas cooled thermal reactors have attracted the attention of researchers towards radiation as a mode of energy transfer. It is worth mentioning that unlike convection and conduction, the radiative heat transfer mechanism is rather more complex. However some reasonable approximations have been found satisfactory to make the radiative system solvable. The works of Sparrow and Cess [22], Howell [23] and Vyas and Rai [24]describe the essentials of the radiative heat transfer. Many other pertinent radiative heat transfer studies for different configurations have also been reported by researchers like Plumb et al. [25], Hossain and Takhar [26], Raptis [27], Sadeek and Salem [28], Al-Odat[29], Prasad et al.[30], Mukopadhyay [31], Vyas and Shrivastava [32], Vyas and Ranjan [33], Chauhan and Kumar [34], Baoku et al. [35], Babu et al. [36], etc.

\section{Problem Formulation}

Let us consider the problem of the radiation effect on the buoyancy induced heat and mass transfer of optically dense viscous incompressible fluid by natural convection over an isothermal slender body of revolution embedded in a saturated porous medium, with a prescribed axial symmetric wall temperature $T_{w}$ which is higher than the ambient temperature $T_{\infty}$ (see Fig.1). Thus, as a result of the buoyancy force, an upward convective fluid movement is induced. The variations of the fluid properties are limited to density variation which affects the buoyancy force term only. The origin of the co-ordinate system is placed at the vertex of the slender body of revolution where $\mathrm{x}$ represents the distance along the body of revolution and $r$ represents the distance normal to the surface of the revolving slender body.

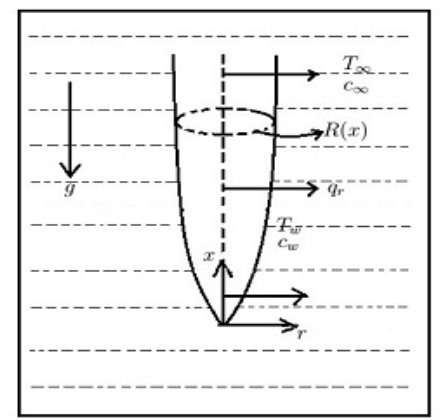

Fig. 1: A slender body of revolution embedded in saturated porous medium

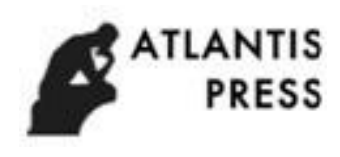


So, the governing equations for the problem under consideration with boundary layer and Boussinesq approximations, Darcy's law and radiative fluid properties are given in cylindrical co-ordinate system as

$$
\begin{gathered}
\frac{\partial}{\partial x}(r u)+\frac{\partial}{\partial r}(r u)=0 \\
\frac{1}{r} \frac{\partial \psi}{\partial r}=\frac{K g}{v}\left(\beta_{T}\left(T-T_{\infty}\right)+\beta_{c}\left(c-c_{\infty}\right)\right) \\
\frac{\partial \psi}{\partial r} \frac{\partial T}{\partial x}-\frac{\partial \psi}{\partial x} \frac{\partial T}{\partial r}=\alpha \frac{\partial}{\partial r}\left(r \frac{\partial T}{\partial r}\right)-\frac{1}{\rho c_{p}} \frac{\partial}{\partial r}\left(r q_{r}\right) \\
\frac{\partial \psi}{\partial r} \frac{\partial c}{\partial x}-\frac{\partial \psi}{\partial x} \frac{\partial c}{\partial r}=D \frac{\partial}{\partial r}\left(r \frac{\partial c}{\partial r}\right) \\
\rho=\rho_{\infty}\left[1-\beta_{T}\left(T-T_{\infty}\right)-\beta_{c}\left(c-c_{\infty}\right)\right]
\end{gathered}
$$

where $u=\frac{1}{r} \frac{\partial \psi}{\partial r}$ and $v=-\frac{1}{r} \frac{\partial \psi}{\partial x}$ are the velocity components along $x$ - and $r$-directions respectively and $\psi$ is the stream function. $\beta_{T}$ and $\beta_{c}$ are the coefficients of thermal and concentration expansion. $K$ is the permeability, $g$ is the acceleration due to gravity, $v$ and $\rho$ are the kinematic viscosity and density of convective fluid. Also, $\alpha$ is the thermal diffusivity of porous medium, $c_{p}$ is the specific heat at constant pressure, $q_{r}$ is the radiative heat flux and $D$ is the diffusivity.

If the transfer process is assumed to occur at low concentration-difference such that the interfacial velocity due to mass diffusion can be neglected, the boundary conditions are,

at the body surface, i.e. at $r=R(x)$,

at infinity, i.e. $r \rightarrow \infty$,

$$
\begin{cases}v=0, & T=T_{w}(x)=T_{\infty}+A x^{a} \\ & c=c_{w}(x)=c_{\infty}+B x^{b}\end{cases}
$$

$$
\begin{cases}v=0, & T=T_{\infty} \\ & c=c_{\infty},\end{cases}
$$

where $\boldsymbol{R}(\boldsymbol{x})$ describes the surface shape of the axi-symmetric body and $\boldsymbol{a}, \boldsymbol{b}, \boldsymbol{A}$ and $\boldsymbol{B}$ are constants. Using the Rosseland approximation for radiation (Brewster [37]), one can write

$$
q_{r}=-\frac{4 \gamma^{*}}{3 k^{*}}\left(\frac{\partial T^{4}}{\partial r}\right)
$$

where $\gamma^{*}$ is the Stefan Boltzmann constant and $k^{*}$ is the absorption coefficient.

Assuming that the temperature difference within the flow is such that $T^{4}$ may be expanded in a Taylor series about $T_{\infty}$, the free stream temperature after neglecting higher order terms can be linearized to obtain

$$
T^{4} \approx 4 T_{\infty}^{3} T-3 T_{\infty}^{4} .
$$

In view of equations (2.8) and (2.9) equation (2.3) reduces to

$$
\frac{\partial \psi}{\partial r} \frac{\partial T}{\partial x}-\frac{\partial \psi}{\partial x} \frac{\partial T}{\partial r}=\alpha\left(1+\frac{4}{3} R_{d}\right) \frac{\partial}{\partial r}\left(r \frac{\partial T}{\partial r}\right)
$$

where $\alpha=\frac{k}{\rho c_{p}}$ is thermal diffusivity of the porous medium and $R_{d}=\frac{4 \gamma^{*} T_{\infty}^{3}}{3 k k^{*}} \quad$ is the radiation parameter ( $k=$ effective thermal conductivity).

To solve the above simultaneous equations, the following dimensionless parameters are introduced:

$$
\begin{array}{ll}
\eta=R_{a}\left(\frac{r}{x}\right)^{2} & ,
\end{array}
$$


where $R_{a}=\frac{K g \beta_{T}\left(T_{w}-T_{\infty}\right) x}{\alpha v}$ is the modified Rayleigh number.

Setting $\eta=\eta_{0}$, where $\eta_{0}$ is a constant and is numerically small for a slender body, equation (2.11) prescribes both the shape and size of the body with its surface given by

$$
R(x)=\left(\frac{v \alpha \eta_{0}}{K g \beta_{T} A}\right)^{\frac{1}{2}} x^{\frac{1-a}{2}}
$$

For problems of practical interest, the value of the constant ' $a$ ' is less than or equal to unity. For instance, the body is circular cylinder when $a=1$, a paraboloid when $a=0$, and a cone when $a=-1$.

After the transformation, the resulting equations are

$$
\begin{gathered}
f^{\prime}=\frac{1}{2}(\theta+N C) \\
\left(1+\frac{4}{3} R_{d}\right) \theta^{\prime \prime}=\frac{1}{2 \eta}\left[a f^{\prime} \theta-(2+f) \theta^{\prime}\right] \\
C^{\prime \prime}=\frac{1}{2 \eta}\left[b \text { Le } f^{\prime} C-(2+\text { Lef }) C^{\prime}\right]
\end{gathered}
$$

with boundary conditions given by

$$
\begin{gathered}
\eta=\eta_{0}, \quad f+(a-1) \eta f^{\prime}=0, \quad \theta=1, \quad C=1 \\
\eta \rightarrow \infty, \quad f^{\prime}=0, \quad \theta=0, \quad C=0,
\end{gathered}
$$

where the parameter $N$ is the buoyancy ratio defined as $N=\frac{\beta_{c}\left(c_{w}-c_{\infty}\right)}{T_{w}-T_{\infty}}$. Thus, $N$ measures the relative importance of mass and thermal diffusion in the buoyancy driven flow. It is clear that $N$ is zero for purely thermal buoyancy driven flow, infinite for mass driven flow, positive for aiding flow and negative for opposing flow.

The prime denotes differentiation with respect to $\eta, \eta \in[0, \infty)$. Here $f(\eta)$ is non-dimensional velocity related to the stream function $\psi(x, y), \theta(\eta)$ and $C(\eta)$ are the dimensionless temperature and concentration profiles. $L e=\frac{\alpha}{D}$ is Lewis number.

In this work, it has been shown that the similarity solutions exist for the case of thermal buoyancy driven flow (i.e. for $N=0$ ). Similarly, it has been shown that equations (2.14)-(2.16) also permit solutions for the important case $a=b=0$ which corresponds to paraboloid with constant temperature and concentration.

\section{Integral Treatment}

The transformed energy equation (2.15) and the constituent mass transfer equation (2.16) can be integrated with respect to $\eta$ from $\eta=\eta_{0}$ to $\eta=\infty$ to obtain from

$$
\begin{aligned}
& -2 \eta_{0} R_{d}^{*} \theta^{\prime}\left(\eta_{0}\right)=\int_{\eta_{0}}^{\infty} f^{\prime} \theta d \eta+2\left(1-R_{d}^{*}\right)+f\left(\eta_{0}\right) \theta\left(\eta_{0}\right) \\
& -2 \eta_{0} C^{\prime}\left(\eta_{0}\right)=L e \int_{\eta_{0}}^{\infty} f^{\prime} C d \eta+f\left(\eta_{0}\right) C\left(\eta_{0}\right) \\
& \text { where } R_{d}^{*}=1+\frac{4}{3} R_{d} .
\end{aligned}
$$

The temperature and concentration profiles are now expressed in terms of the following exponential functions :

$$
\begin{gathered}
\theta(\eta)=\exp \left\{-\left(\frac{\eta-\eta_{0}}{\varepsilon_{\tau}}\right)\right\} \\
C(\eta)=\exp \left\{-\xi\left(\frac{\eta-\eta_{0}}{\varepsilon_{\tau}}\right)\right\}
\end{gathered}
$$

which satisfy the boundary conditions (2.17) and (2.18). Here $\delta_{T}$ is the arbitrary scale for thermal boundary layer thickness, while $\xi$ is its ratio to the concentration boundary layer thickness. Using the relations (3.3) and (3.4), the equations (3.1) and (3.2) by taking into account the relation (2.14), reduce to two distinct expressions for $\delta_{T}$ as

$$
\begin{gathered}
{\left[\frac{1}{2}+\frac{N}{\xi+1}\right] \delta_{T}^{2}-\left[\eta_{0}(N+1)+4\left(1-R_{d}^{*}\right)\right] \delta_{T}-4 \eta_{0} R_{d}^{*}=0} \\
L e\left[\frac{1}{\xi+1}+\frac{N}{2 \xi}\right] \delta_{T}^{2}-L e\left[\eta_{0}(N+1)\right] \delta_{T}-4 \eta_{0} \xi=0 .
\end{gathered}
$$

The above equations govern the thermal boundary layer thickness for the coupled heat and mass transfer by natural convection from a vertical cylinder embedded in a saturated porous medium.

From equations (3.5) and (3.6), we obtain 
where

$$
\delta_{T}=\frac{A_{4} \xi^{3}+A_{5} \xi^{2}+A_{6} \xi+A_{7}}{-A_{3} \xi^{2}+A_{8} \xi+A_{9}}
$$

$A_{1}=\eta_{0}(N+1)+4\left(1-R_{d}^{*}\right), A_{2}=4 \eta_{0} R_{d}^{*}, A_{3}=\operatorname{Le}\left[\eta_{0}(N+1)\right], A_{4}=4 \eta_{0}$,

$A_{5}=(1+2 N) A_{4}, A_{6}=-A_{2} L e(N+2), A_{7}=-A_{2} N L e$,

$A_{8}=A_{1} L e(N+2)-A_{3}(1+2 N)$ and $A_{9}=A_{1} N$ Le .

The equations (3.5) and (3.6) can be combined to obtain the algebraic equation

where

$$
\alpha_{7} \xi^{7}+\alpha_{6} \xi^{6}+\alpha_{5} \xi^{5}+\alpha_{4} \xi^{4}+\alpha_{3} \xi^{3}+\alpha_{2} \xi^{2}+\alpha_{1} \xi+\alpha_{0}=0
$$

$\alpha_{0}=A_{7}^{2}(1+2 N)-2 A_{1} A_{7} A_{9}-A_{2} A_{9}^{2}$,

$\alpha_{1}=A_{7}^{2}+2 A_{6} A_{7}(1+2 N)-2 A_{1} A_{7} A_{9}-2 A_{1} A_{6} A_{9}-2 A_{1} A_{7} A_{8}-2 A_{2} A_{8} A_{9}$,

$\alpha_{2}=2 A_{6} A_{7}+A_{6}^{2}(1+2 N)+2 A_{5} A_{7}(1+2 N)-2 A_{1} A_{6} A_{9}-2 A_{1} A_{7} A_{8}-2 A_{1} A_{5} A_{9}-2 A_{1} A_{6} A_{8}+$

$2 A_{1} A_{3} A_{7}+2 A_{2} A_{3} A_{9}-A_{2} A_{8}^{2}$,

$\alpha_{3}=A_{6}^{2}+2 A_{5} A_{7}+2 A_{4} A_{7}(1+2 N)+2 A_{5} A_{6}(1+2 N)-2 A_{1} A_{5} A_{8}-2 A_{1} A_{5} A_{9}-2 A_{1} A_{6} A_{8}-2 A_{1} A_{4} A_{9}+$ $2 A_{1} A_{3} A_{6}+2 A_{2} A_{3} A_{8}+2 A_{1} A_{3} A_{7}$

$\alpha_{4}=2 A_{4} A_{7}+A_{5}^{2}(1+2 N)+2 A_{4} A_{7}(1+2 N)+2 A_{5} A_{6}-2 A_{1} A_{4} A_{9}-2 A_{1} A_{5} A_{8}-2 A_{1} A_{4} A_{8}+2 A_{1} A_{3} A_{6}+$ $2 A_{2} A_{3} A_{8}$

$\alpha_{5}=A_{5}^{2}+2 A_{4} A_{6}+2 A_{4} A_{5}(1+2 N)-2 A_{1} A_{4} A_{8}+2 A_{1} A_{3} A_{5}+2 A_{1} A_{3} A_{4}$,

$\alpha_{6}=2 A_{4} A_{5}+A_{4}^{2}(1+2 N)+2 A_{1} A_{3} A_{4}$ and $\alpha_{7}=A_{4}^{2}$.

The value of $\xi$ is determined from equation (3.8) by using computer software MATHEMATICA.

As $\xi$ is determined from equation (3.8), the local Nusselt and local Sherwood numbers, which are of maininterest in terms of heat and mass transfer respectively, are given as

$$
\begin{aligned}
N u & =\frac{h x}{k}=\frac{-\left.\left(\frac{d T}{d x}\right)\right|_{r=R(x)}}{T_{w}-T_{\infty}}=-2 \eta_{0} \theta^{\prime}\left(\eta_{0}\right)(R a)^{\frac{1}{2}} \\
N u & =\frac{2 \eta_{0}}{\delta_{T}}(R a)^{\frac{1}{2}} \quad[\text { from Eq.(3.3)] }
\end{aligned}
$$

and

$$
\begin{gathered}
S h=\frac{m x}{D\left(c_{w}-c_{\infty}\right)}=-2 \eta_{0} C^{\prime}\left(\eta_{0}\right)(R a)^{\frac{1}{2}} \\
S h=2 \eta_{0} \frac{\xi}{\delta_{T}}(R a)^{\frac{1}{2}}
\end{gathered}
$$

[from Eq.(3.4)]

where $\delta_{T}$ is given by expression (3.7).

Here our calculations are restricted to the specific case of $\eta_{0}=0.01$ only.

and

$$
\therefore \quad \frac{N u}{(R a)^{\frac{1}{2}}}=\frac{2(0.01)}{\delta_{T}}=(0.02)\left(\frac{1}{\delta_{T}}\right)
$$

$$
\frac{S h}{(R a)^{\frac{1}{2}}}=2(0.01)\left(\frac{\xi}{\delta_{T}}\right)=(0.02)\left(\frac{\xi}{\delta_{T}}\right)
$$

The accuracy acquired in the foregoing approximate expressions may be examined by comparing the approximate heat and mass transfer results against the similarity solution [10] in case of $R_{d}^{*}=1$ for two limiting cases of pure thermal driven flow (i.e. $N=0$ ) and pure mass driven flow (i.e. $N \rightarrow \infty$ ), as follows :

$$
\begin{aligned}
& \frac{N u}{(L e)^{\frac{1}{2}}(R a)^{\frac{1}{2}}}=\frac{S h}{(L e)^{\frac{1}{2}}(R a)^{\frac{1}{2}}}=0.018: \text { similarity and integral solutions } \\
& \frac{N u}{(L e)^{\frac{1}{2}}(R a)^{\frac{1}{2}}}=\frac{S h}{(L e)^{\frac{1}{2}}(R a)^{\frac{1}{2}}}=0.020: \text { approximate }
\end{aligned}
$$

Our approximate expression given by equations (3.9) and (3.10) tend to overestimate heat and mas transfer rates under these physical limiting conditions. It is not unusual to have anerror of 10 percent or more, depending on the assumed profile. However, this situation can be remedied by adjusting the multiplicative constant, namely, replacing 0.020 by 0.018 . Thus, we propose the following final approximate formulae :

$$
\begin{aligned}
& \frac{N u}{(R a)^{\frac{1}{2}}}=0.018\left(\frac{1}{\delta_{T}}\right) \\
& \frac{S h}{(R a)^{\frac{1}{2}}}=0.018\left(\frac{\xi}{\delta_{T}}\right)
\end{aligned}
$$


where the boundary layer thickness ratio $\xi$ is given by equation (3.8)

Table1.Variation of local Nusselt and local Sherwood numbers for $R_{d}^{*}=1.4$

\begin{tabular}{|c|c|c|c|c|c|c|c|}
\hline$N$ & Le & $\frac{N u}{(R a)^{\frac{1}{2}}}$ & $\frac{S h}{(R a)^{\frac{1}{2}}}$ & $N$ & Le & $\frac{N u}{(R a)^{\frac{1}{2}}}$ & $\frac{S h}{(R a)^{\frac{1}{2}}}$ \\
\hline \multirow[t]{5}{*}{-1} & 1 & 0.004779 & 0.058933 & \multirow[t]{5}{*}{4} & 1 & 0.007638 & 0.144815 \\
\hline & 4 & 0.005169 & 0.122622 & & 4 & 0.006473 & 0.270644 \\
\hline & 10 & 0.005328 & 0.196870 & & 10 & 0.006120 & 0.394637 \\
\hline & 50 & 0.005488 & 0.446858 & & 50 & 0.005825 & 0.678956 \\
\hline & 100 & 0.005527 & 0.634239 & & 100 & 0.005765 & 0.802726 \\
\hline \multirow[t]{5}{*}{0} & 1 & 0.005589 & 0.085427 & \multirow[t]{5}{*}{10} & 1 & 0.010603 & 0.1982 \\
\hline & 4 & 0.005589 & 0.169265 & & 4 & 0.007416 & 0.35501 \\
\hline & 10 & 0.005589 & 0.261505 & & 10 & 0.006658 & 0.495479 \\
\hline & 50 & 0.005589 & 0.533848 & & 50 & 0.006102 & 0.758757 \\
\hline & 100 & 0.005589 & 0.703914 & & 100 & 0.006004 & 0.846576 \\
\hline \multirow[t]{5}{*}{1} & 1 & 0.006174 & 0.104370 & & & & \\
\hline & 4 & 0.005868 & 0.202319 & & & & \\
\hline & 10 & 0.005760 & 0.306205 & & & & \\
\hline & 50 & 0.005661 & 0.587893 & & & & \\
\hline & 100 & 0.005639 & 0.743497 & & & & \\
\hline
\end{tabular}

Table2. Variation of local Nusselt and local Sherwood numbers for $L e=1$

\begin{tabular}{|c|c|c|c|c|c|c|c|}
\hline$N$ & $R_{d}^{*}$ & $\frac{N u}{(R a)^{\frac{1}{2}}}$ & $\frac{S h}{(R a)^{\frac{1}{2}}}$ & $N$ & $R_{d}^{*}$ & $\frac{N u}{(R a)^{\frac{1}{2}}}$ & $\frac{S h}{(R a)^{\frac{1}{2}}}$ \\
\hline \multirow[t]{5}{*}{0} & 2 & 0.002244 & 0.086745 & \multirow[t]{5}{*}{6} & 2 & 0.002627 & 0.16484 \\
\hline & 4 & 0.000749 & 0.087419 & & 4 & 0.000788 & 0.164883 \\
\hline & 6 & 0.000449 & 0.087561 & & 6 & 0.000463 & 0.164903 \\
\hline & 8 & 0.000321 & 0.087622 & & 8 & 0.000328 & 0.164912 \\
\hline & 10 & 0.000250 & 0.087657 & & 10 & 0.000254 & 0.164917 \\
\hline \multirow[t]{5}{*}{1} & 2 & 0.002336 & 0.105158 & \multirow[t]{5}{*}{8} & 2 & 0.002718 & 0.181970 \\
\hline & 4 & 0.000759 & 0.105587 & & 4 & 0.000796 & 0.181983 \\
\hline & 6 & 0.000453 & 0.105678 & & 6 & 0.000466 & 0.181992 \\
\hline & 8 & 0.000323 & 0.105718 & & 8 & 0.000330 & 0.181997 \\
\hline & 10 & 0.000251 & 0.10574 & & 10 & 0.000255 & 0.1820 \\
\hline \multirow[t]{5}{*}{2} & 2 & 0.002409 & 0.120249 & \multirow[t]{5}{*}{10} & 2 & 0.002804 & 0.197162 \\
\hline & 4 & 0.000767 & 0.120543 & & 4 & 0.000803 & 0.197079 \\
\hline & 6 & 0.000456 & 0.120608 & & 6 & 0.000468 & 0.19708 \\
\hline & 8 & 0.000324 & 0.120636 & & 8 & 0.000331 & 0.197081 \\
\hline & 10 & 0.000252 & 0.120652 & & 10 & 0.000256 & 0.197083 \\
\hline
\end{tabular}

Table3. Variation of local Nusselt and local Sherwood numbers for $N=1$

\begin{tabular}{|c|c|c|c|c|c|c|c|}
\hline$L e$ & $R_{d}^{*}$ & $\frac{N u}{(R a)^{\frac{1}{2}}}$ & $\frac{S h}{(R a)^{\frac{1}{2}}}$ & $L e$ & $R_{d}^{*}$ & $\frac{N u}{(R a)^{\frac{1}{2}}}$ & $\frac{S h}{(R a)^{\frac{1}{2}}}$ \\
\hline \multirow[t]{5}{*}{1} & 2 & 0.002336 & 0.105158 & \multirow[t]{5}{*}{20} & 2 & 0.002263 & 0.410816 \\
\hline & 4 & 0.0007594 & 0.105587 & & 4 & 0.0007524 & 0.410938 \\
\hline & 6 & 0.0004533 & 0.105678 & & 6 & 0.0004505 & 0.410994 \\
\hline & 8 & 0.0003231 & 0.105718 & & 8 & 0.0003217 & 0.411022 \\
\hline & 10 & 0.0002510 & 0.10574 & & 10 & 0.0002501 & 0.411036 \\
\hline \multirow[t]{5}{*}{4} & 2 & 0.002289 & 0.002289 & \multirow[t]{5}{*}{40} & 2 & 0.002257 & 0.5398 \\
\hline & 4 & 0.0007542 & 0.202978 & & 4 & 0.0007508 & 0.539874 \\
\hline & 6 & 0.0004515 & 0.203058 & & 6 & 0.0004503 & 0.539913 \\
\hline & 8 & 0.0003222 & 0.203094 & & 8 & 0.0003215 & 0.539934 \\
\hline & 10 & 0.0002504 & 0.203114 & & 10 & 0.0002500 & 0.539949 \\
\hline 10 & 2 & 0.002272 & 0.306042 & 50 & 2 & 0.002255 & 0.586223 \\
\hline
\end{tabular}




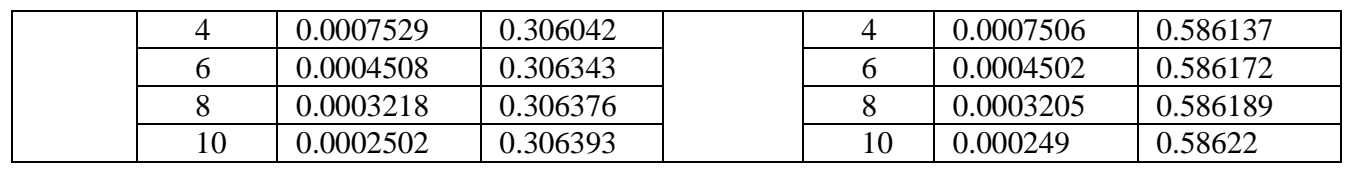

Table4. Variation of local Nusselt and local Sherwood numbers for $N=-1$

\begin{tabular}{|c|c|c|c|c|c|c|c|}
\hline Le & $R_{d}^{*}$ & $\frac{N u}{(R a)^{\frac{1}{2}}}$ & $\frac{S h}{(R a)^{\frac{1}{2}}}$ & Le & $R_{d}^{*}$ & $\frac{N u}{(R a)^{\frac{1}{2}}}$ & $\frac{S h}{(R a)^{\frac{1}{2}}}$ \\
\hline \multirow[t]{5}{*}{1} & 2 & 0.002101 & 0.061574 & \multirow[t]{5}{*}{20} & 2 & 0.002215 & 0.282731 \\
\hline & 4 & 0.0007327 & 0.062919 & & 4 & 0.0007460 & 0.283936 \\
\hline & 6 & 0.0004437 & 0.063203 & & 6 & 0.0004485 & 0.284199 \\
\hline & 8 & 0.0003182 & 0.063327 & & 8 & 0.0003207 & 0.284313 \\
\hline & 10 & 0.0002480 & 0.063395 & & 10 & 0.0002495 & 0.284377 \\
\hline \multirow[t]{5}{*}{4} & 2 & 0.002173 & 0.125239 & \multirow[t]{5}{*}{40} & 2 & 0.002225 & 0.400757 \\
\hline & 4 & 0.0007412 & 0.126571 & & 4 & 0.0007472 & 0.401856 \\
\hline & 6 & 0.0004468 & 0.126851 & & 6 & 0.0004489 & 0.402103 \\
\hline & 8 & 0.0003198 & 0.126972 & & 8 & 0.0003209 & 0.402211 \\
\hline & 10 & 0.0002490 & 0.12704 & & 10 & 0.0002496 & 0.402274 \\
\hline \multirow[t]{5}{*}{10} & 2 & 0.002201 & 0.199278 & \multirow[t]{5}{*}{50} & 2 & 0.002227 & 0.448321 \\
\hline & 4 & 0.0007444 & 0.200556 & & 4 & 0.0007475 & 0.449377 \\
\hline & 6 & 0.0004479 & 0.200828 & & 6 & 0.0004491 & 0.449618 \\
\hline & 8 & 0.0003204 & 0.200946 & & 8 & 0.0003210 & 0.449726 \\
\hline & 10 & 0.0002493 & 0.201012 & & 10 & 0.0002497 & 0.449785 \\
\hline
\end{tabular}

\section{Results and discussion}

The numerical values of the local Nusselt and local Sherwood numbers have been computed in Tables 1-4. From Table 1, it is obvious that for a fixed value of the radiation parameter $R_{d}^{*}\left(R_{d}^{*}=1.4\right)$, the values of the local Nusselt and local Sherwood numbers increase alongwith the increasing values of buoyancy ratio $N$ in case of aiding flow $(N>0)$. Although both the local numbers show increasing trend alongwith $N$, but this trend ofincrease in local Sherwood number is more pronounced as compared to that of the local Nusselt number.

Also, for $N=0$ and $R_{d}^{*}=1.4$, the local Nusselt number exhibits a constancy in its trend for increasing values of Lewis number $(L e)$. This trend is also clear from the Figs. 4 and 5. However for $N>0$, the local Nusselt number shows a decreasing trend and the local Sherwood number an increasing trend along with increasing values of $L e$.

It is also evident from Table 1 that for opposing flow $(N<0)$, both the local Nusselt and local Sherwood numbers show an increasing trend alongwith the increasing values of $L e$ in case of opposing flow ( i.e. for $N=-1)$ and for $R_{d}^{*}=1.4$. This trend is also explainable from Figs. 2 and 3.

From Table 2, it is observed that for different values of $N(N=0,1,2,6,8,10)$ and for $L e=1$, the local Nusselt number shows a decreasing trend along with the increasing values of the radiation parameter $R_{d}^{*}$, where as the local Sherwood number shows an increasing trend. But this effect of radiation parameter on local Nusselt and local Sherwood numbers is considerably small.

From Table 3, it is also observed that for a fixed value of $N$ (i:e: for $N=1$ ) and for different values of $L e(L e=1 ; 4 ; 10 ; 20 ; 40 ; 50)$, the local Nusselt number shows a decreasing trend alongwith increasing values of the radiation parameter $R_{d}^{*}$, whereas the local Sherwood number shows an increasing trend. But these trends of decrease in the local Nusselt number or increase in local Sherwood number are very small, thereby implying that the radiation effects do not play signicant role in governing the characteristics pertaining to temperature and concentration of the flow-field.

From Table 4, for the opposing flow (i.e. for $N<0$ ), the local Nusselt number shows a decreasing trend for a fixed value of $L e$ and for increasing values of $R_{d}^{*}$. On the contrary, the local Sherwood number shows an increasing trend for increasing values of $R_{d}^{*}$. Moreover, both the local Nusselt and Sherwood numbers exhibit an increasing trend for increasing values of $L e$ and for fixed values of $R_{d}^{*}$. 


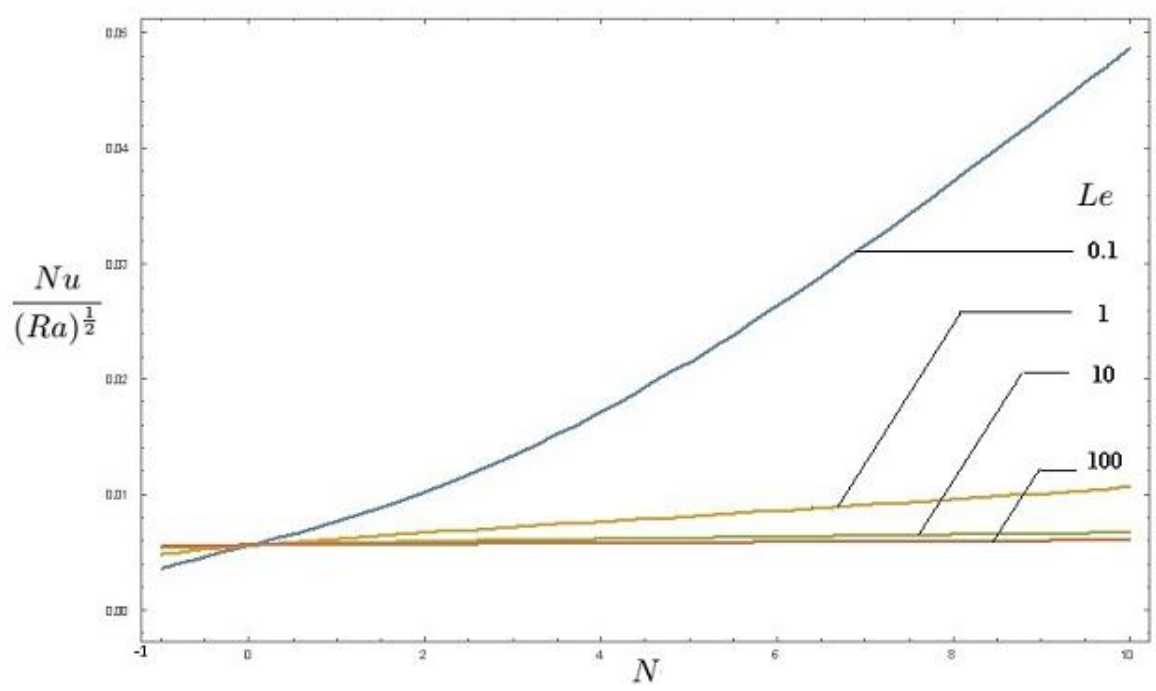

Fig.2. Heat transfer coefficient as a function of buoyancy ratio for $R_{d}^{*}=1.4$

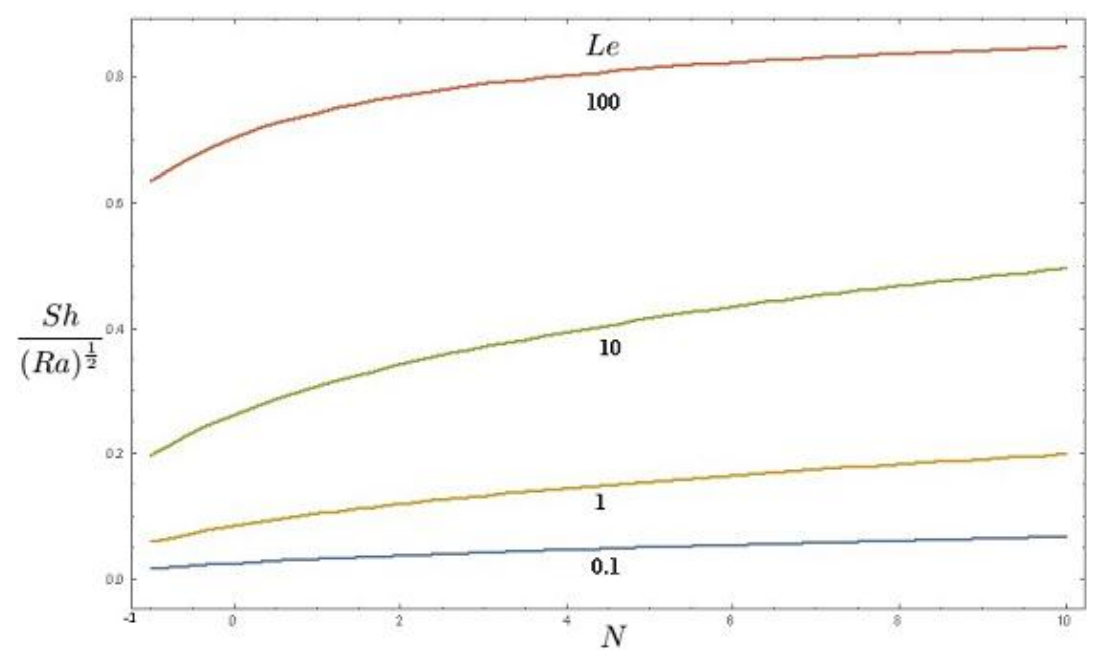

Fig.3. Mass transfer coefficient as a function of buoyancy ratio for $R_{d}^{*}=1.4$

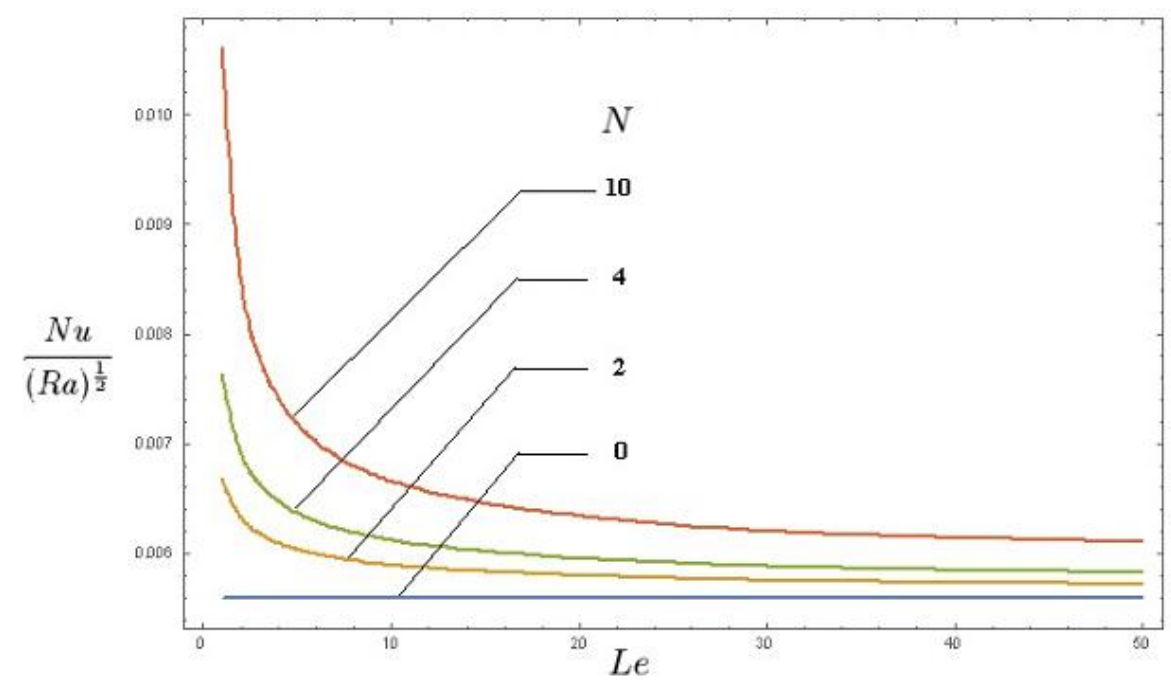

Fig.4. Heat transfer coefficient as a function of the Lewis number for $R_{d}^{*}=1.4$ 


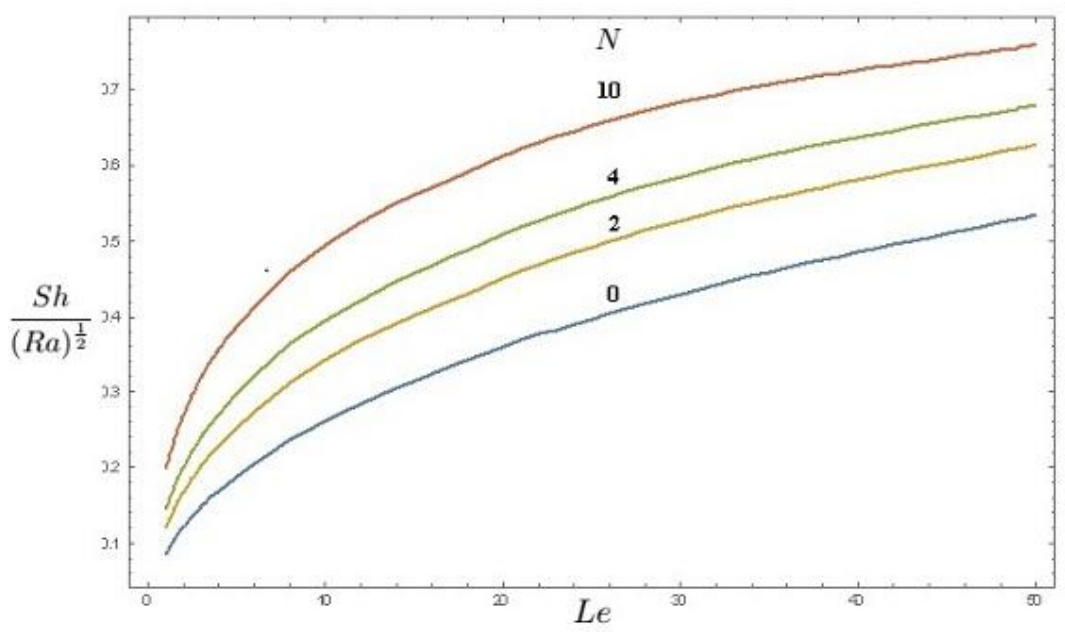

Fig.5. Mass transfer coefficient as a function of the Lewis number for $R_{d}^{*}=1.4$

\section{Concluding remarks}

For the coupled heat and mass transfer by natural convection in a porous medium, the solutions have been presented for the important case of radiating paraboloid with constant temperature and concentration. The governing parameters for the problem under consideration are the Lewis number $L e$, buoyancy ratio $N$ and the radiation parameter $R_{d}^{*}$. From the numerical and graphical results, it is observed that the Lewis number has a more pronounced effect on the concentration field than it does on the temperature field. The radiative fluid properties past the paraboloid have also been taken into consideration. It has been found that although the radiation parameter affects both the temperature and concentration fields, the effects are very meagre in terms of numerical values. Also, the results obtained in this study for $R_{d}^{*}$ are in close agreement graphically with the corresponding results of Lai et al.[10] who tackled the problem in the absence of radiation effect by using shooting technique in conjunction with Runge-Kutta fourth order method. Finally, the integral approach of Von Karman type adopted in this problem is very efficient and handy for making use of for engineering applications. Thus, the integral treatment remains a powerful means to attack boundary layer problems, since it naturally captures correct asymptotic behaviours as compared to the scale arguments which are not completely free from the risk of misinterpretation.

\section{Acknowledgement}

One of the authors, Shaikh Nasreen Bano, would like to express her sense of gratitude to Dr. Babasaheb Ambedkar Technological University, Lonere for providing the TEQIP-II fellowship for pursuing her Ph.D. programme.

\section{References}

[1] Bejan, A., Khair, K.R. : Heat and mass transfer by natural convection in a porous medium. Int. J. Heat Mass Transfer . 28, 909 (1985)

[2] Merkin, J.H. : Free convection boundary layers on axi-symmetric and two-dimensional bodies of arbitrary shape in a saturated porous medium. Int.J. Heat Mass Transfer. 22, 1461(1979)

[3] Nakayama, A., Koyama, H. : Free convective heat transfer over a non-isothermal body of arbitrary shape embedded in a fluid-saturated porous medium. J. Heat Transfer. 109, 125(1987)

[4] Nield, D.A. : Onset of thermohaline convection in a porous medium. Water Resour. Res. 4, 553(1968)

[5] Khan,A.A., Zebib, A.: Double diffusive instability in a vertical layer of a porous medium. J. Heat Transfer. 103, 179(1981)

[6] Trevisan, O.V., Bejan, A. : Natural convection with combined heat and mass transfer buoyancy effects in a porous medium. Int. J. Heat Mass Transfer. 28, 1597(1985)

[7] Trevisan, O.V., Bejan, A. : Mass and heat transfer by natural convection in a vertical slot filled with porous medium. Int. J. Heat Mass Transfer. 29, 403(1986)

[8] Trevisan, O.V., Bejan, A.: Mass and heat transfer by high Rayleigh number convection in a porous medium heated from below. Int. J. Heat Mass Transfer. 30, 2341(1985) 
[9] Yücel, A. : Heat and mass transfer about vertical surfaces in saturated porous media. AIChE Symposium Series. 269, Vol. 85, 344(1989)

[10] Lai, F. C., Choi, C. Y., Kulacki, F. A. : Coupled heat and mass transfer by natural convection from slender bodies of revolution in porous media. Int. Commu. Heat Mass Transfer. 17, 609(1990)

[11] Lai, F. C., Kulacki, F. A. : Heat and mass transfer by natural convection from vertical surfaces in porous media. Int. J. Heat Mass Transfer 34, 1189(1991)

[12] Nakayama, A., Hossain, M. A. : An integral treatment for combined heat and mass transfer by natural convection in a porous medium. Int. J. Heat Mass Transfer 38, 761(1995)

[13] Singh, P., Queeny : Free convection heat and mass transfer along a vertical surface in a porous medium. Acta Mechanica. 123, 69(1997)

[14] Angirasa, D., Peterson, G. P., Pop, I. : Combined heat and mass transfer by natural convection with opposing buoyancy effect in a fluid saturated porous medium. Int. J. Heat Mass Transfer. 40, 2755(1997)

[15] Amahmid, A., Hasnaoui, M., Mamou, M., Vasseur, P., Boundary layer flows in a vertical porous enclosure induced by opposing buoyancy forces. Int. J. Heat Mass Transfer 42, 3599(1999)

[16] Yih, K. A. : Coupled heat and mass transfer by free convection over a truncated cone in porous media. Acta Mechanica. 137, 83(1999)

[17] Chamkha, A. J.: Coupled heat and mass transfer by natural convection about a truncated cone in the presence of magnetic field and radiation effects. Num. Heat Transfer 39(A), 511(2001)

[18] Bansod, V. J., Singh, P., Rathishkumar, B. V. : Heat and mass transfer by natural convection from a vertical surface to the stratied Darcian fluid. J. Porous Media. 5(1), 57(2002)

[19] Bansod, V. J. : The Darcy model of the boundary layer flows in a horizontal porous medium. J. Porous Media. 6(4), 273(2003)

[20] Singh, B. B., Chandarki, I.M. : Integral treatment of coupled heat and mass transfer by natural convection from a cylinder in porous media. Int. Commu. Heat Mass Transfer. 36, 269(2009)

[21] Singh, P., Sharma, K. : Integral method to free convection in thermally stratied porous medium. Acta Mechanica. 83, 157(1990)

[22] Sparrow, E. M., Cess, R. D. : Radiation heat transfer Brooke/Cole. Belmont, California, (1970)

[23] Howell, J. R. : Radiation transfer in porous media, In : Vafai K. (ed.). CRC Press, New York, (2000)

[24] Vyas, P., Rai, A. : Radiative fluid properties flow due to a point sink inside a cone filled with porous medium. Applied Mathematical Science 6(87), 4307(2012)

[25] O. A. Plumb, J. S. Huenefeld and E. J. Eschbach, IThe effect of cross flow and radiation on natural convection from vertical heated surfaces in saturated porous media. AIAA $16^{\text {th }}$ Thermophysics Conference, Polo Alto, CA, USA, (1981)

[26] Hossain, M.A., Takhar, H.S. : Radiation effect on mixed convection along a vertical plate with uniform surface temperature. Heat Mass Transfer 31(4), 243(1996)

[27] Raptis,A.: Radiation and free convection flow through a porous medium. Int.Commu. Heat Mass Transfer. 25(2), 289(1998)

[28] Sedeek, M.A., Salem, A.M. : Laminar mixed convection adjacent to vertical continuously stretching sheet with variable viscosity and variable thermal diffusivity. Heat Mass Transfer. 41(12), 1048(2005)

[29] Al-Odat, M.Q., Al-Hussein, F.M.S., Damesh, R.A. : Influence of radiation on mixed convection over a wedge in non-Darcy porous medium. Forschung Ingenieurwesen 69(4), 209(2005)

[30] Prasad, V.R., Reddy, N.B., Muthucumarswamy, R. : Radiation and mass transfer effects on twodimensional flow past an impulsively started infinite vertical plate. Int. J. Thermal Sci. 46(2), 1251(2007)

[31] Mukopadhyay,S.: Effects of radiation and variable fluid viscosity on flow and heat transfer along a symmetric wedge. J. Appld. Fluid Mech. 2(2), 29(2009)

[32] Vyas, P., Shrivastava, N. : Radiative MHD flow over a non-isothermal stretching sheet in a porous medium. Appl. Mathematical Sci. 4(50), 2475(2010)

[33] Vyas,P., Ranjan, A. : Dissipative MHD boundary layer flow in a porous medium over a sheet stretching non-linearly in the presence of radiation. Appl. Mathematical Sci. 4(61-64), 3133(2010)

[34] Chauhan,D.S., Kumar,V. : Radiation effects on mixed convection flow and viscous heating in a vertical channel partially filled with a porous medium. Tamkang J. Science and Engineering. 14(2), 97(2011)

[35] Baoku, I.G., Israel- Cookey ,C., Olajuwan,B.I. : Influence of thermal radiation on a transient MHD Coutte flow through a porous medium. J. Appl. Fluid Mech. 5(1), 641(2014)

[36] Babu, P. R., Rao, J.A., Sheri, S. : Radiation effect on MHD heat and mass transfer flow over a shrinking sheet with mass suction. J. Appl. Fluid Mech. 7(4), 641(2014)

[37] Brewster, M.Q. : Thermal Radiative transfer and properties. John Wiley and Sons, New York (1992). 